\title{
ComplexLeader: um jogo para o desenvolvimento de liderança com base na complexidade
}

\author{
Mauricio C. Lopes ${ }^{1}$, João P. S. Gonçalves ${ }^{1}$, Francisco A. P. Fialho², Christianne C. \\ S. R. Coelho ${ }^{2}$ \\ ${ }^{1}$ Departamento de Sistemas e Computação - Universidade Regional de Blumenau
} (FURB)

Rua Antonio da Veiga, 140 - 80030-903 - Blumenau - SC - Brasil

${ }^{2}$ Departamento de Engenharia de Conhecimento - Universidade Federal de Santa Catarina - Florianópolis - SC - Brasil

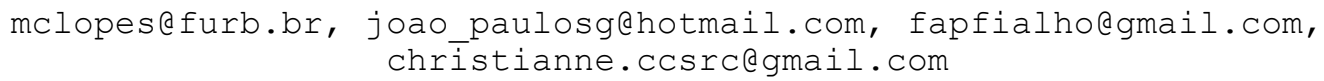

Abstract. This article aims to present the Electronic Business Game (EBG) ComplexLeader for leadership development. The ComplexLeader was developed considering the theoretical and methodological assumptions of ComplexView framework that is based on Complex Adaptive Systems (CAS) and Agent-Based Modeling (ABM). In its beta version the ComplexLeader was applied in two groups which indicated need for improvement in the model and readjustment in communication with its users, bringing it closer to a more business language.

Resumo. O presente artigo tem por objetivo apresentar o Jogo de Empresas Eletrônico (JEE) ComplexLeader para o desenvolvimento de liderança. $O$ ComplexLeader foi desenvolvido considerando os pressupostos teóricos e metodológicos do framework ComplexView que têm, entre suas bases, conhecimentos de Sistemas Adaptativos Complexo e Modelagem Baseada em Agentes. Em sua versão beta o ComplexLeader foi aplicado em duas turmas o que indicou necessidade de melhorias no modelo e readequação na comunicação com seus usuários, aproximando-a de uma linguagem mais empresarial.

\section{Introdução}

O método educacional baseado em Jogos de Empresas Eletrônicos (JEEs) tem sido reconhecido como eficaz e positivo para a aprendizagem (FARIA e WELLINGTON, 2005; FORTMULLER, 2009). O sucesso dos JEEs está relacionado à sua utilização no desenvolvimento de práticas gerenciais envolvendo competências e habilidades principalmente em tarefas relacionadas à gestão estratégica, gestão de produção, gestão de mercado e gestão financeira (FARIA e WELLINGTON, 2004). Entretanto, ao tratar dos processos e relações sociais nas organizações, os JEEs ainda oferecem muitas oportunidades para terem seu aproveitamento ampliado e sua efetividade melhorada. 


\section{CBIE-LACLO 2015}

Anais dos Workshops do IV Congresso Brasileiro de Informática na Educação (CBIE 2015)

Um destes processos, de particular interesse no presente artigo, é o desenvolvimento de liderança.

O conceito do que vem a ser liderança tem evoluído e se adaptado à dinâmica das novas exigências sociais (DAY, 2001; MCCRIMMON, 2005), mas ainda falta consenso sobre ele (ALDRICH, 2003; KILBURG e DONOHUE, 2011). Em geral, as práticas gerenciais estão associadas às questões burocráticas das organizações e a liderança está associada à mudança (KOTTER, 1990). O desenvolvimento de liderança tem sofrido impacto da mudança de paradigma da sociedade industrial para a sociedade do conhecimento, gerando um novo conjunto de demandas e necessidades para a liderança nas organizações do conhecimento (ALDRICH, 2003; NONAKA, TOYAMA e HIRATA, 2008; UHL-BIEN, MARION e MCKELVEY, 2007).

A Era do Conhecimento agrega novos desafios às organizações, ao tratar de mercados mais abertos e globalizados, ao inserir novos elementos culturais e tecnológicos, e exigir diferentes formas e modelos de organizações e sistemas de produção e mercado (YUKL, 2006). Na Era Industrial as atividades eram tratadas de modo mais orientado e linear. Os fenômenos eram estudados e analisados isoladamente através de relações diretas de causa e efeito (NORTHOUSE, 2003). Na Era do Conhecimento, surgem novos valores e dinâmicas, sem se centrar apenas em uma única pessoa. As equipes passam a ser mais valorizadas e as abordagens tornam-se mais incertas e menos determinísticas.

$\mathrm{Na}$ Era Industrial os líderes também tinham um maior número de tarefas e preocupações de ordem gerencial (HANSSON e MONSTED, 2008; MINTZBERG, 1998). Crescentes desafios fizeram com que novos elementos fossem agregados às suas responsabilidades, distanciando-os da ação gerencial e posicionando-os mais próximos de atividades adaptativas e de inovação (UHL-BIEN, MARION e MCKELVEY, 2007). Desta forma, a bibliografia sobre liderança cresceu com base em diferentes abordagens e múltiplas dimensões (AVOLIO, WALUMBWA e WEBER, 2009).

Uma destas abordagens é a Teoria da Liderança com base na Complexidade (TLC) que se fundamenta na visão dos Sistemas Complexos Adaptativos (SCA) estudados na Ciência da Complexidade (UHL-BIEN, MARION e MCKELVEY, 2007; UHL-BIEN e MARION, 2009). A Complexidade estuda o comportamento coletivo de muitas unidades que interagem entre si, com o potencial de evoluir ao longo do tempo (COVENEY e HIGHFIELD, 1995). Com base nos fundamentos e nos modelos de simulação da Complexidade é possível reproduzir o comportamento dos sistemas reais, o que proporciona a criação de laboratórios virtuais de experimentação para que se possam fazer previsões sobre o comportamento dos sistemas em situações complexas (ZHANG e LEEZER, 2010). A abordagem da Complexidade tem sido utilizada para estudar os fenômenos das ciências naturais, e sua aplicação no estudo de fenômenos sociais tem grande potencial a ser explorado (ZHANG e LEEZER, 2010). Esta possibilidade abre também novas perspectivas para o método educacional baseado em JEEs, sobretudo para tratar de temas e abordagens interdisciplinares, na medida em que a complexidade não trata um problema a partir da perspectiva de uma única disciplina.

Os JEEs têm sido utilizados como método de ensino desde o fim da década de 50, no século XX (FARIA et al., 2009). Usados a partir de diferentes perspectivas e finalidades, eles são aplicados às mais diferentes áreas. Os JEEs oferecem mecanismos 
para que os participantes aprendam a lidar, na prática, com os problemas reais existentes nas organizações. Eles proporcionam aos participantes atividades de planejamento estratégico, resolução de problemas, trabalho em equipe, visão organizacional, entre outras (ANDERSON e LAWTON, 2009; FARIA et al., 2009; LOPES, NIVEIROS e FIALHO, 2011; LAINEMA, 2009)

No sentido de tornar o processo de produção de JEEs ágil e orientado às necessidades atuais da formação em liderança, Lopes (2011) propôs o framework ComplexView que tem por finalidade apoiar a produção de JEEs voltados ao desenvolvimento de liderança, com base nos fundamentos da Complexidade. Com base neste framework o presente artigo tem por objetivo apresentar o JEE ComplexLeader desenvolvido para preparar lideranças para lidarem com os desafios da sociedade do conhecimento. O JEE proposto combina teorias aderentes e consistentes com os fundamentos da TLC e simula questões relacionadas às forças emergentes (condições habilitadoras e dinâmicas da complexidade) e resultados adaptativos, considerando o desenvolvimento dos indivíduos e dos grupos.

\section{Framework ComplexView}

O framework ComplexView (LOPES, 2011) tem, entre seus propósitos, lançar os fundamentos para a criação de uma fábrica de JEEs, com base em conceitos da Teoria da Complexidade. Com base nele espera-se ter um processo mais rápido e efetivo para a criação de novos JEEs. O framework ComplexView está organizado em cinco níveis, conforme apresentado na Figura 1.

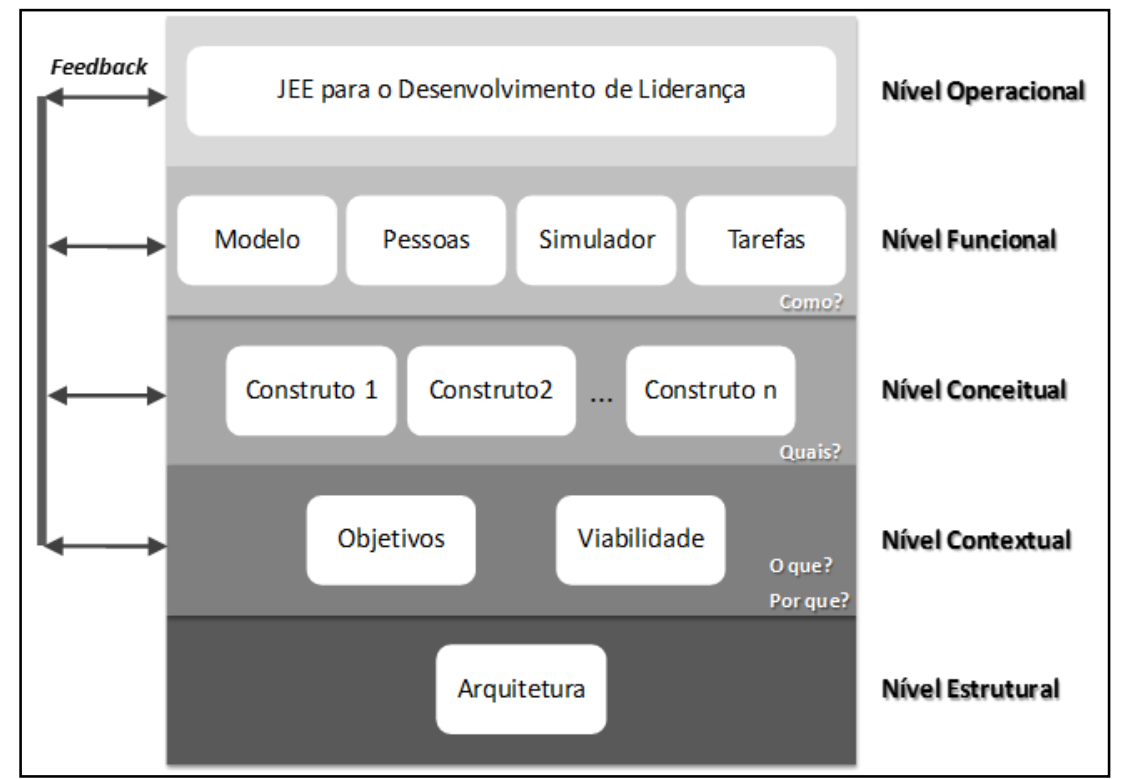

Fonte: Lopes (2011)

Figura 1 - Estrutura do Framework ComplexView

Lopes (2011) destaca uma visão geral sobre cada um dos níveis:

a) nível estrutural: define a arquitetura comum para todos os JEEs produzidos com base no framework. Esta arquitetura está associada à visão de mundo e às teorias que sustentam o framework; 


\section{CBIE-LACLO 2015}

Anais dos Workshops do IV Congresso Brasileiro de Informática na Educação (CBIE 2015)

b) nível contextual: estuda a viabilidade e define os objetivos de um JEE específico a ser produzido com base no ComplexView. A viabilidade deve responder à questão sobre por que desenvolver o JEE e os objetivos devem definir o que será feito;

c) nível conceitual: define os construtos teóricos utilizados para a modelagem de um JEE específico. Os construtos apontam quais as teorias e modelos serão utilizados para atender os objetivos;

d) nível funcional: descreve o processo de produção do JEE, considerando os componentes apresentados na Figura 4 (seção 2.3). Este processo define como desenvolver o JEE com base na arquitetura (nível estrutural), na viabilidade e nos objetivos (nível contextual), e nos construtos (nível conceitual);

e) nível operacional: descreve o JEE produzido, considerando as escolhas e caminhos seguidos nos níveis conceitual e funcional.

\section{Jogo de Empresas ComplexLeader}

O ComplexLeader foi desenvolvido com base nos níveis propostos pelo ComplexView. No nível estrutural o JEE ComplexLeader seguiu o modelo proposto pelo framework (Figura 2) que é baseado em Sistemas Complexos Adaptativos (SCA) e Modelagem Baseada em Agentes (MBA).

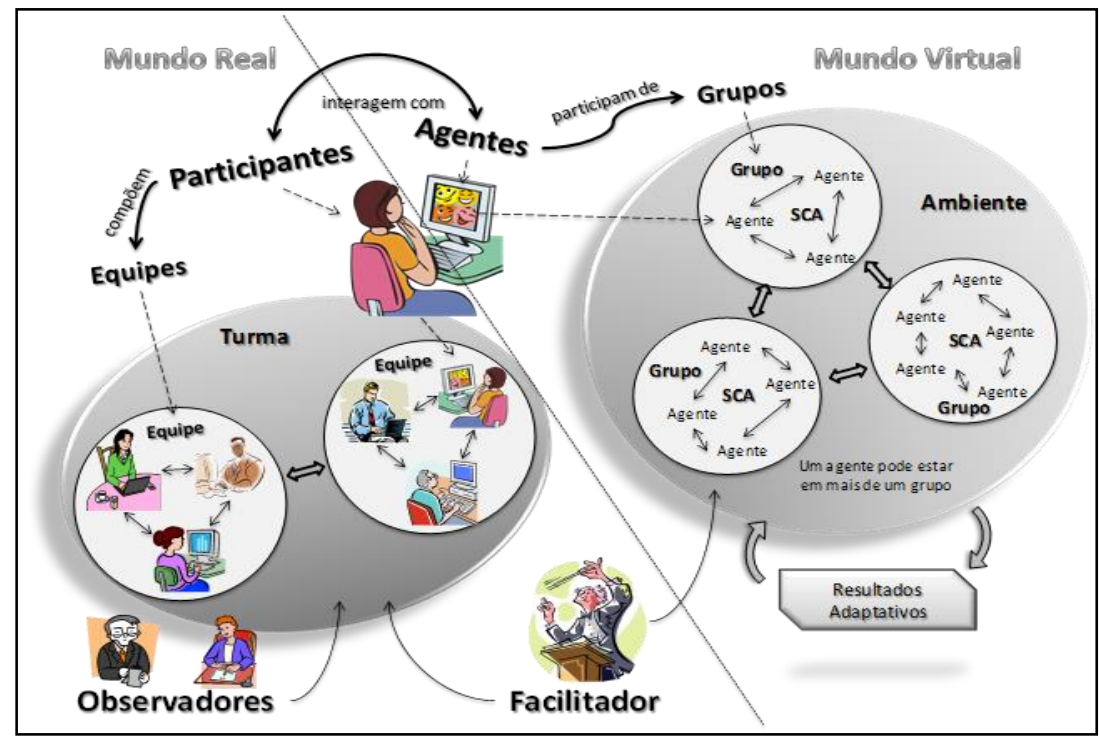

Fonte: Lopes (2011, p. 238).

Figura 2 - Nível Estrutural do ComplexLeader

No ComplexLeader os jogadores (participantes) são organizados em equipes. Cada equipe assume uma organização que tem um determinado número de pessoas (agentes). As pessoas organizam-se em grupos para executar projetos. Os objetivos de aprendizagem definidos para o jogo estão descritos na Tabela 1. Os objetivos são definidos no nível contextual do framework. 


\section{Tabela 1 - Objetivos de Aprendizagem do ComplexLeader}

a) estimular a reflexão sobre aspectos teórico-conceituais de liderança;

b) enfatizar o capital humano e social como elemento estratégico na gestão das organizações;

c) compreender os fenômenos da liderança sob a visão da complexidade;

d) entender as dinâmicas formais e informais dos processos de liderança;

e) exercitar práticas de liderança de modo a ampliar as competências e habilidades pessoais e promover a mudança de atitude nos processos de liderança;

f) analisar a inserção das necessidades motivacionais e do nível de prontidão em um ambiente complexo;

g) avaliar o perfil de aptidões cerebrais e seus efeitos em grupos de alto desempenho;

h) entender as implicações do modelo de meta-aprendizagem no contexto de sistemas complexos adaptativos;

i) praticar a gestão de sistemas complexos adaptativos com base nas necessidades motivacionais, níveis de prontidão, aptidões cerebrais e modelo de meta aprendizagem.

Fonte: Lopes (2011, p. 240).

Os construtos teóricos que fundamentam o ComplexLeader estão apresentados na Tabela 2. Os construtos são a base do nível conceitual do framework.

Tabela 2 - Construtos Teóricos do ComplexLeader

\begin{tabular}{|c|c|c|}
\hline Construto & Autor (es) & Justificativa \\
\hline $\begin{array}{l}\text { Hierarquia } \\
\text { Necessidades }\end{array}$ & Maslow ([196-]) & $\begin{array}{l}\text { Utilizada parcialmente. Define as necessidades das } \\
\text { pessoas virtuais (agentes). Foram modeladas apenas as } \\
\text { necessidades motivacionais (social, estima e realização). }\end{array}$ \\
\hline $\begin{array}{l}\text { Liderança } \\
\text { Situacional }\end{array}$ & $\begin{array}{l}\text { Hersey, Blanchard e } \\
\text { Johnson (2007) }\end{array}$ & $\begin{array}{l}\text { Utilizada parcialmente. Foram modelados apenas os } \\
\text { conceitos de maturidade no trabalho e maturidade } \\
\text { psicológica da teoria. }\end{array}$ \\
\hline Aptidões Cerebrais & Miranda (1997) & Define o perfil de aptidões cerebrais das pessoas. \\
\hline $\begin{array}{l}\text { Modelo Meta } \\
\text { Learning }\end{array}$ & Losada e Heaphy (2004) & $\begin{array}{l}\text { Utilizada parcialmente. Define as relações interpessoais } \\
\text { necessárias para modelar grupos. }\end{array}$ \\
\hline $\begin{array}{l}\text { Teoria da Liderança } \\
\text { com base na } \\
\text { Complexidade }\end{array}$ & $\begin{array}{l}\text { Uhl-Bien, Marion e } \\
\text { McKelvey (2007) e Uhl- } \\
\text { Bien e Marion (2009) }\end{array}$ & $\begin{array}{l}\text { É a base conceitual do CompexLeader. Modela as } \\
\text { relações de liderança que produzem emergência e } \\
\text { adaptação na organização. }\end{array}$ \\
\hline
\end{tabular}

Fonte: Lopes (2011, p. 244).

O processo de produção do ComplexLeader seguiu as diretrizes e métodos propostos no nível funcional do framework (Figura 3).

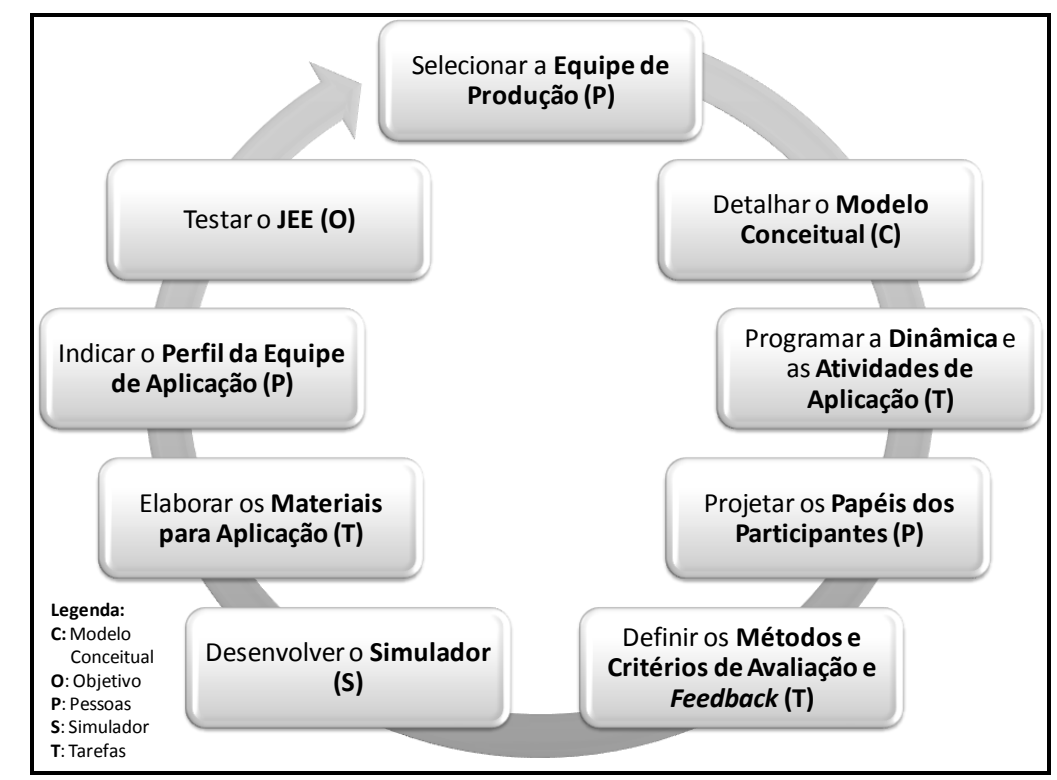

Fonte: Lopes (2011, p. 201).

Figura 3 - Etapas do Nível Funcional 


\section{CBIE-LACLO 2015}

Anais dos Workshops do IV Congresso Brasileiro de Informática na Educação (CBIE 2015)

Dentre as etapas propostas no nível funcional algumas merecem destaque. No detalhamento do modelo conceitual foi desenvolvido um modelo matemático de relações entre os construtos teóricos apresentados na Tabela 2. Foram modeladas três entidades: organização, projetos e pessoas. Os jogadores tomam decisões e acompanham os resultados sobre cada uma destas entidades.

As decisões sobre a organização envolvem festas comemorativas, horário flexível de trabalho, intervalo de descanso e salas de lazer. Os resultados são acompanhados com base em diversos indicadores entre os quais destacam-se o surgimento espontâneo de novos projetos, a quantidade de projetos concluídos com sucesso, a margem financeira (receitas - despesas), as forças emergentes que geram resultados positivos, o nível de prontidão das pessoas e o espaço emocional dos projetos. A interface computacional contendo alguns elementos das decisões e resultados sobre a organização é apresentada na Figura 4.

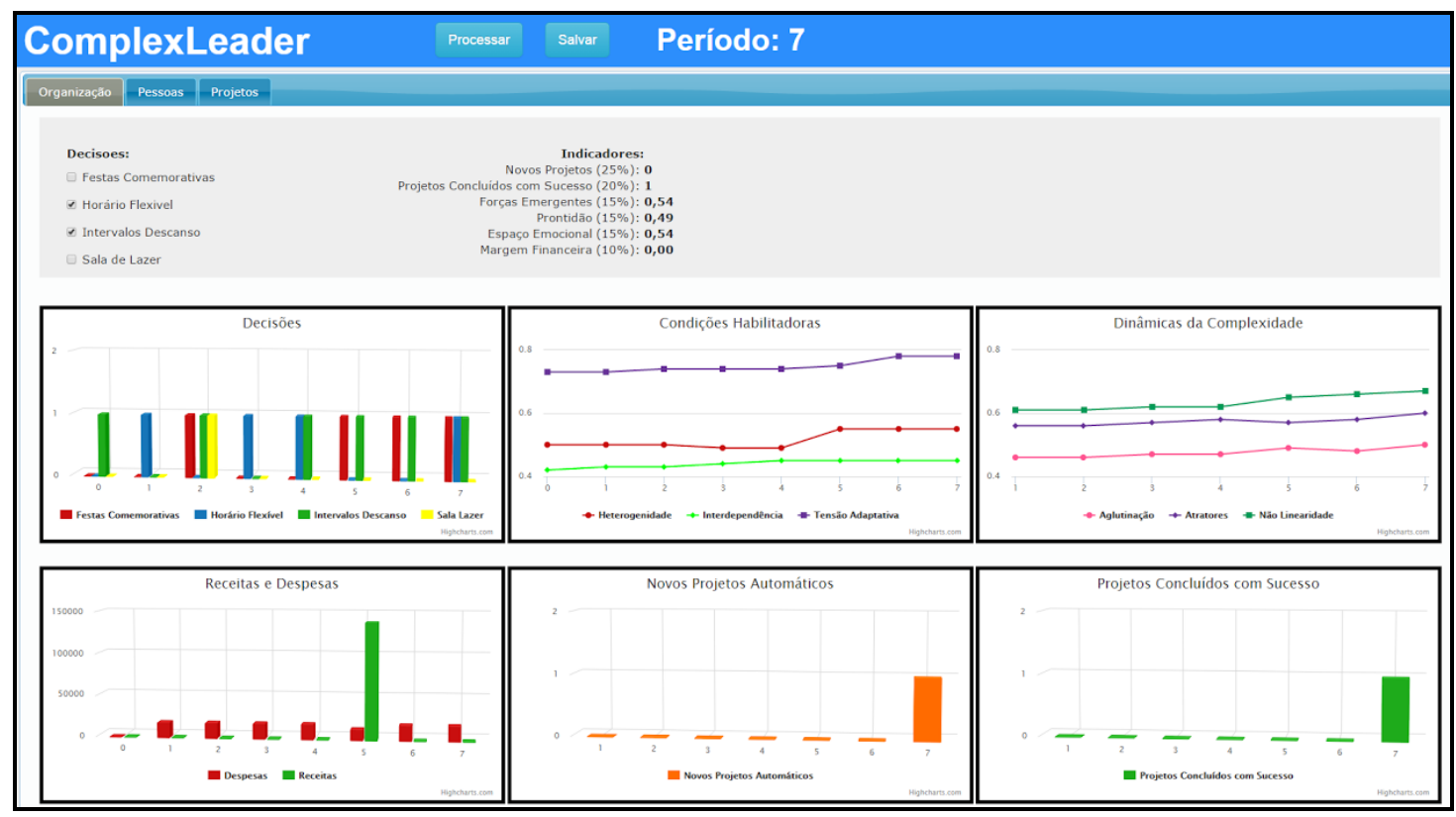

Figura 4 - Decisões e Resultados da Organização no ComplexLeader

As decisões sobre as pessoas envolvem três tipos de treinamento definidos como estratégias de desenvolvimento pessoal, além da possibilidade de se oferecer prêmios e salários individualizados. As decisões tomadas pelos participantes sobre as pessoas influenciam suas aptidões cerebrais, seu nível de maturidade e sua motivação e prontidão resultantes do processamento matemático do jogo, definido no detalhamento do nível conceitual. Para cada pessoa são geradas despesas associadas aos salários e investimentos realizados. As pessoas têm também afinidades associadas às outras, em função de interesses pessoais ou do ambiente de trabalho. A interface computacional contendo alguns elementos das decisões e resultados sobre as pessoas é apresentada na Figura 5. 
CBIE-LACLO 2015

Anais dos Workshops do IV Congresso Brasileiro de Informática na Educação (CBIE 2015)

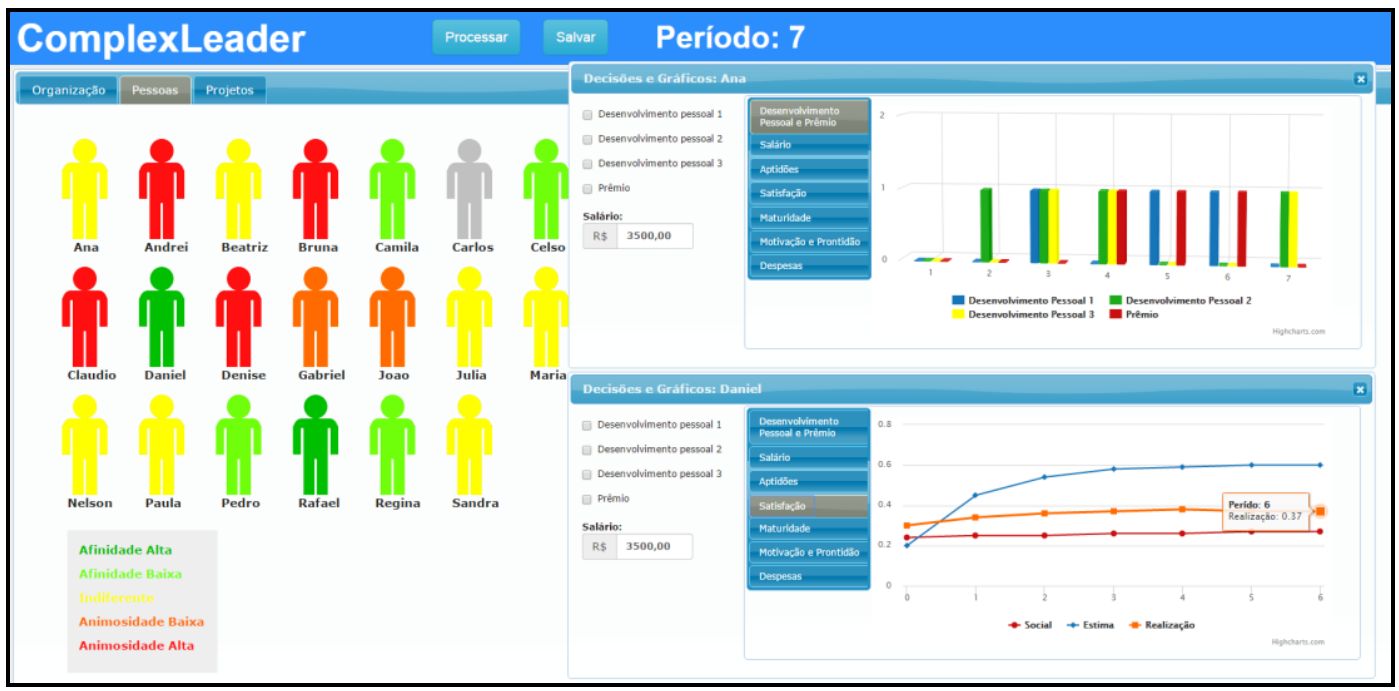

Figura 5 - Decisões e Resultados das Pessoas no ComplexLeader

Quanto aos projetos, as decisões envolvem o estilo de acompanhamento do líder e o investimento feito para o desenvolvimento do trabalho em equipe. Os projetos são de diferentes tipos: inovação, aprendizagem e adaptabilidade. Eles têm um período máximo para sua conclusão e uma receita potencial associada. Os participantes do jogo têm como propósito conseguir que os projetos sejam concluídos com sucesso para que a receita potencial de cada um deles seja efetivada como receita da organização. Para isto eles precisam alocar as pessoas nos projetos de acordo com o perfil de ambos. A cada período é possível acompanhar o andamento dos projetos, o espaço emocional do grupo, e a situação das condições habilitadoras e dinâmicas da complexidade do projeto, bem como acompanhar suas despesas. Caso o projeto chegue ao final sem ser concluído, o mesmo é desfeito e a organização contabiliza os custos gerados. As pessoas também podem propor projetos os quais devem ser aceitos ou não pelos participantes do jogo. A interface computacional contendo alguns elementos das decisões e resultados sobre as pessoas é apresentada na Figura 6.

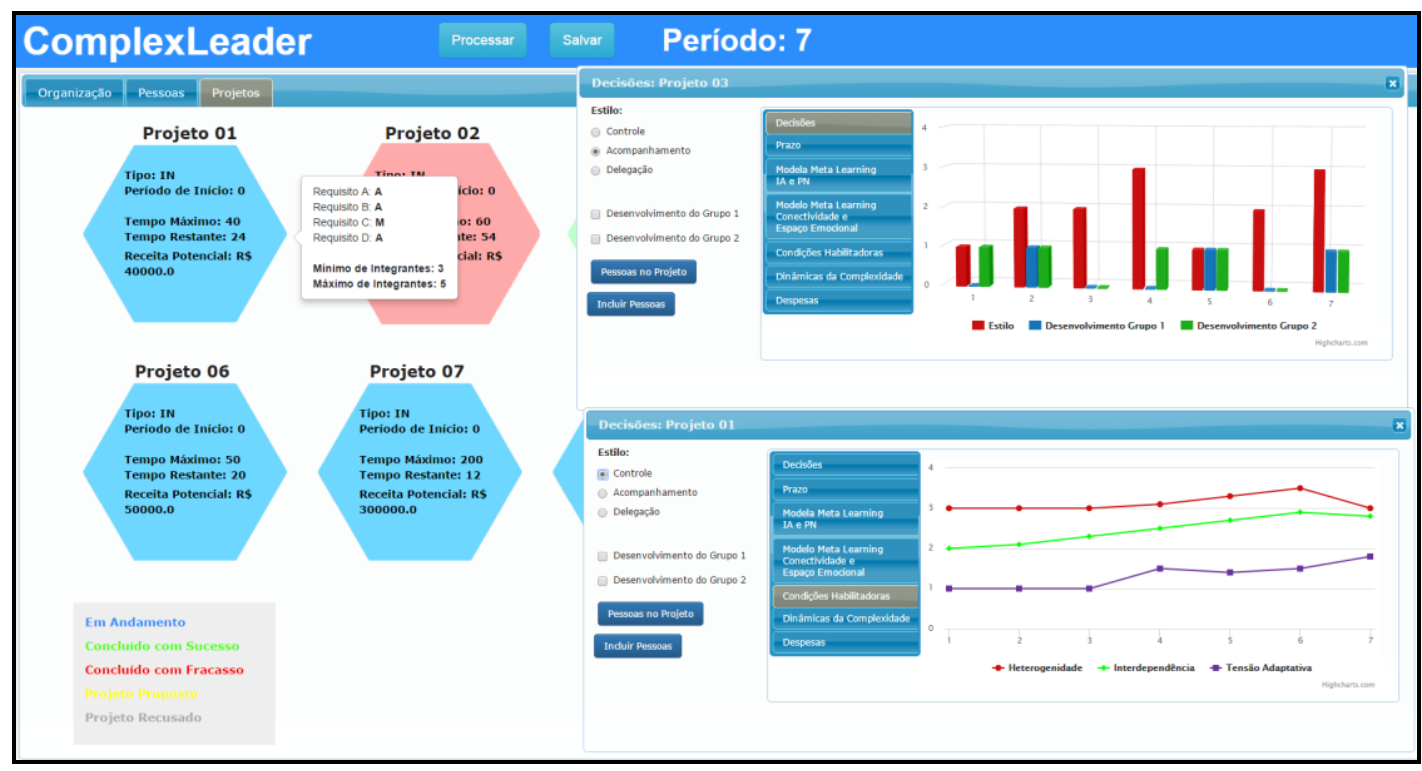

Figura 6 - Decisões e Resultados dos Projetos no ComplexLeader 


\section{CBIE-LACLO 2015}

Anais dos Workshops do IV Congresso Brasileiro de Informática na Educação (CBIE 2015)

Outro destaque no nível funcional é a dinâmica do jogo (Figura 7). As interações dos participantes não dependem de uma sequência fixa de atividades. O processamento é contínuo e as decisões ocorrem em um ambiente de mudanças constantes. A configuração básica do ComplexLeader define que a cada cinco minutos as decisões tomadas são submetidas ao modelo matemático gerando novos resultados. Com esta dinâmica espera-se que o JEE seja mais dinâmico e próximo da realidade, promova maior interação e envolvimento dos participantes, ao contrário dos modelos tradicionais de JEEs cujo modelo de processamento é linear e exige maior flexibilidade dos participantes para acompanhar diversos acontecimentos no jogo de forma simultânea (LOPES, 2011).

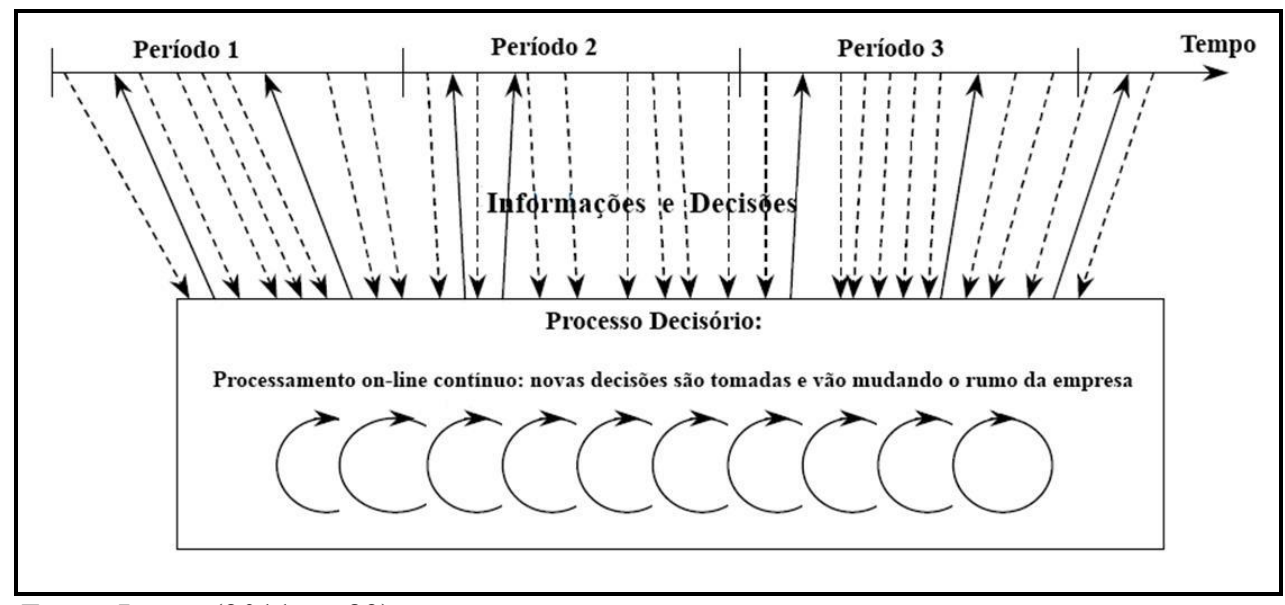

Fonte: Lopes (2011, p. 83).

Figura 7 - Dinâmica do ComplexLeader

\section{Considerações Finais}

O desenvolvimento do JEE ComplexLeader foi fundamental para validar as etapas do framework ComplexView. O jogo foi incialmente criado em uma versão preliminar (beta) na linguagem Delphi, que foi aplicada duas vezes em ambientes monousuário. A primeira aplicação ocorreu em um grupo de estudantes e professores de Mestrado e Doutorado, apenas para teste. A segunda foi aplicada com um grupo de estudantes de Mestrado já como instrumento de avaliação dos estudantes. A versão web, cuja interface foi apresentada no presente artigo, está em fase de conclusão e apresenta melhoramentos em relação à versão beta do JEE ComplexLeader. Os resultados das aplicações foram promissores, mas revelaram alguns aspectos a serem considerados e discutidos.

$\mathrm{O}$ primeiro trata da modelagem matemática. As aplicações revelaram que o modelo apresenta uma tendência de convergência nos resultados, independente das decisões, após um número significativo de processamentos. Isto deverá ser solucionado com ajustes tanto nas decisões quanto nas variáveis intervenientes em cada aspecto do modelo. O segundo trata da distância entre a linguagem conceitual do jogo da que é reconhecido nas organizações, uma vez que o jogo apresenta conceitos da Complexidade que não são totalmente dominados em ambientes educacionais onde o tema não seja tratado em profundidade, assim como são em grande parte desconhecidos em ambientes empresariais ou não educacionais. Isto deverá ser solucionado ampliando as explicações sobre os conceitos e inserindo exemplos de situações cotidianos. O 


\section{CBIE-LACLO 2015}

Anais dos Workshops do IV Congresso Brasileiro de Informática na Educação (CBIE 2015)

terceiro trata de aspectos de avaliação educacional sobre o conteúdo tratado no jogo. Uma vez que se usa o jogo ao longo de uma disciplina (trimestral ou semestral), isto deverá ser solucionado trabalhando conteúdos teóricos juntos com a sua aplicação, tornando a aprendizagem mais significativa.

O desenvolvimento de JEE baseados na Complexidade tem grande potencial de uso interdisciplinar, uma vez que esta área do conhecimento tem este princípio em si mesma. Especificamente, o ComplexLeader trata do desenvolvimento de habilidades de liderança que tem entre suas bases conhecimentos da Psicologia, Administração, Antropologia, Filosofia, entre outros, modelados com conhecimentos da Matemática e da Computação. O framework ComplexView, por exemplo, tem entre suas bases conhecimentos sobre Sistemas Complexos Adaptativos e Sistemas Multiagentes. O ComplexLeader demonstra ser possível unir conhecimentos de diversas áreas de modo a gerar aprendizagem aos seus participantes em aspectos fundamentais para $o$ desenvolvimento da sociedade do conhecimento.

\section{Referências}

ALDRICH, C. The new core of leadership. T and D, v. 57, n. 3, p. 32-37, 2003.

ANDERSON, P. H.; LAWTON, L. Business simulations and cognitive learning: developments, desires, and future directions. Simulation \& Gaming, v. 40, n. 2, p. 193-216, 2009.

AVOLIO, B. J.; WALUMBWA, F. O.; WEBER, T. J. Leadership: current theories, research, and future directions. Annual Review of Psychology, v. 60, p. 421-449, 2009. doi: 10.1146/annurev.psych.60.110707.163621.

COVENEY, P.; HIGHFIELD, R. Frontiers of complexity: the search for order in a chaotic world. New York (NY): Ballantine Books, 1995.

DAY, D. V. Leadership development: a review in context. The Leadership Quarterly, v. 11, n. 4, p. 581-613, 2001.

FARIA, A. J.; HUTCHINSON, D.; WELLINGTON, W. J.; GOLD, S. Developments in business gaming: a review of the past 40 years. Simulation \& Gaming, v. 40, n. 4, p. 464-487, 2009.

FARIA, A. J.; WELLINGTON, W. J. A survey of simulation game users, former-users, and never-users. Simulation \& Gaming, v. 35, p. 178-207, 2004.

FARIA, A. J.; WELLINGTON, W. J. Validating business gaming: business game conformity with pims findings. Simulation \& Gaming, v. 36, n. 2, p. 259-273, 2005.

FORTMULLER, R. Learning through business games: acquiring competences within virtual realities. Simulation \& Gaming, v. 40, n. 1, p. 68-83, 2009.

HANSSON, F.; MONSTED, M. Research leadership as entrepreneurial organizing for research. Higher Education, v. 55, n. 6, p. 651-670, 2008.

HERSEY, P.; BLANCHARD, K.; JOHNSON, D. E. Management of organizational behavior: leading human resources. 9a. ed. Upper Saddle River (NJ): Prentice-Hall, 2007. 


\section{CBIE-LACLO 2015}

Anais dos Workshops do IV Congresso Brasileiro de Informática na Educação (CBIE 2015)

KILBURG, R. R.; DONOHUE, M. D. Toward a "grand unifying theory" of leadership: implications for consulting psychology. Consulting Psychology Journal: Practice and Research, v. 63, n. 1, p. 6-25, 2011. doi:10.1037/a0023053.

KOTTER, J. Force for change: how leadership differs from management. New York, NY: Free Press, 1990.

LAINEMA, T. Perspective making: constructivism as a meaning-making structure for simulation gaming. Simulation \& Gaming, v. 40, n. 1, p. 48-67, 2009.

LOPES, M. C. ComplexView: um framework para a produção de jogos de empresas aplicados ao desenvolvimento de ldierança com base na complexidade. Tese de Doutorado (Programa de Pós-Graduação em Engenharia e Gestão do Conhecimento). Departamento de Engenharia do Conhecimento. Universidade Federal de Santa Catarina. Florianópolis (SC), p. 502. 2011.

LOPES, M. C.; NIVEIROS, S.; FIALHO, F. A. P. Jogos de empresas na educação: um método inovador de ensino aprendizagem. In: YOUSSEF, Y. A.; RAMIREZ, A. Social software in higher education: pedagogical models and universities strategies. Palhoça (SC): Editora Unisul, 2011. p. 239-294.

LOSADA, M.; HEAPHY, E. The role of positivity and connectivity in the performance of business teams: a nonlinear dynamics model. American Behavioral Scientist, v. 47, n. 6, p. 740-765, 2004. doi: 10.1177/0002764203260208.

MASLOW, A. H. Introdução a psicologia do ser. Rio de Janeiro (RJ): Eldorado, [196-].

MCCRIMMON, M. Thought leadership: a radical departure from traditional; positional leadership. Management Decision, v. 43, p. 1064-1070, ago/set 2005.

MINTZBERG, H. Covert leadership: notes on managing professionals. Harvard Business Review, v. 36, n. 6, p. 140-147, 1998.

MIRANDA, R. L. Além da inteligência emocional: uso integral das aptidões cerebrais. Rio de Janeiro, RJ: Campus, 1997.

NONAKA, I.; TOYAMA, R.; HIRATA, T. Managing flow: a process theory of the knowledg-based firm. New York (NY): Palgrave Macmillan, 2008.

NORTHOUSE, P. G. Leadership: theory and practice. 3a. ed. Thousand Oaks, California: Sage, 2003.

UHL-BIEN, M.; MARION, R. Complexity leadership in bureaucratic forms of organizing: a meso model. The Leadership Quarterly, v. 20, p. 631-650, 2009.

UHL-BIEN, M.; MARION, R.; MCKELVEY, B. Complexity Leadership Theory: Shifting leadership from the industrial age to the knowledge era. The Leadership Quarterly, v. 18, n. 4, p. 298-318, 2007.

YUKL, G. Leadership in organizations. Upper Saddle River, NJ: Pearson/Prentice Hall, 2006.

ZHANG, Y.; LEEZER, J. Simulating human-like decisions in a memory-based agent model. Computational and Mathematical Organization Theory, v. 16, n. 4, p. 373399, 2010. doi: 10.1007/s10588-010-9077-z. 